\title{
The simplicial volume of hyperbolic manifolds with geodesic boundary
}

\author{
ROBERTO FRIGERIO \\ CRistina Pagliantini
}

\begin{abstract}
Let $n \geq 3$, let $M$ be an orientable complete finite-volume hyperbolic $n$-manifold with compact (possibly empty) geodesic boundary, and let $\operatorname{Vol}(M)$ and $\|M\|$ be the Riemannian volume and the simplicial volume of $M$. A celebrated result by Gromov and Thurston states that if $\partial M=\varnothing$ then $\operatorname{Vol}(M) /\|M\|=v_{n}$, where $v_{n}$ is the volume of the regular ideal geodesic $n$-simplex in hyperbolic $n$-space. On the contrary, Jungreis and Kuessner proved that if $\partial M \neq \varnothing$ then $\operatorname{Vol}(M) /\|M\|<v_{n}$.

We prove here that for every $\eta>0$ there exists $k>0$ (only depending on $\eta$ and $n$ ) such that if $\operatorname{Vol}(\partial M) / \operatorname{Vol}(M) \leq k$, then $\operatorname{Vol}(M) /\|M\| \geq v_{n}-\eta$. As a consequence we show that for every $\eta>0$ there exists a compact orientable hyperbolic $n$-manifold $M$ with nonempty geodesic boundary such that $\operatorname{Vol}(M) /\|M\| \geq v_{n}-\eta$. Our argument also works in the case of empty boundary, thus providing a somewhat new proof of the proportionality principle for noncompact finite-volume hyperbolic $n$-manifolds without geodesic boundary.
\end{abstract}

$53 \mathrm{C} 23 ; 57 \mathrm{~N} 16,57 \mathrm{~N} 65$

\section{Preliminaries and statements}

Let $X$ be a topological space, let $Y \subseteq X$ be a (possibly empty) subspace of $X$, and let $R$ be a ring (in the present paper only the cases $R=\mathbb{R}$ and $R=\mathbb{Z}$ will be considered). For $i \in \mathbb{N}$ we denote by $C_{i}(X ; R)$ the module of singular $i$-chains over $R$, ie the $R$-module freely generated by the set $S_{i}(X)$ of singular $i$-simplices with values in $X$. The natural inclusion of $Y$ in $X$ induces an inclusion of $C_{i}(Y ; R)$ into $C_{i}(X ; R)$, so it makes sense to define $C_{i}(X, Y ; R)$ as the quotient space $C_{i}(X ; R) / C_{i}(Y ; R)$ (of course, if $Y=\varnothing$ we get $C_{i}(X, Y ; R)=C_{i}(X ; R)$ ). The usual differential of the complex $C_{*}(X ; R)$ defines a differential $d_{*}: C_{*}(X, Y ; R) \rightarrow C_{*-1}(X, Y ; R)$. The homology of the resulting complex is the usual relative singular homology of the topological pair $(X, Y)$ and will be denoted by $H_{*}(X, Y ; R)$. 
In what follows, we will denote simply by $C_{i}(X), C_{i}(X, Y)$ respectively the modules $C_{i}(X ; \mathbb{R}), C_{i}(X, Y ; \mathbb{R})$. The $\mathbb{R}$-vector space $C_{i}(X, Y)$ can be endowed with the following natural $L^{1}$-norm: if $\alpha \in C_{i}(X, Y)$, then

$$
\|\alpha\|=\|\alpha\|_{1}=\inf \left\{\sum_{\sigma \in S_{i}(X)}\left|a_{\sigma}\right| \text {, where } \alpha=\left[\sum_{\sigma \in S_{i}(X)} a_{\sigma} \sigma\right] \text { in } C_{i}(X) / C_{i}(Y)\right\} \text {. }
$$

Such a norm descends to a seminorm on $H_{*}(X, Y)$, which is defined as follows: if $[\alpha] \in H_{i}(X, Y)$, then

$$
\|[\alpha]\|=\inf \left\{\|\beta\|, \beta \in C_{i}(X, Y), d \beta=0,[\beta]=[\alpha]\right\}
$$

(note that such a seminorm can be null on nonzero elements of $H_{*}(X, Y)$ ).

\subsection{Simplicial volume}

Throughout the whole paper, every manifold is assumed to be connected and orientable. If $M$ is a compact $n$-manifold with (possibly empty) boundary $\partial M$, then we denote by $[M]_{\mathbb{Z}}$ a generator of $H_{n}(M, \partial M ; \mathbb{Z}) \cong \mathbb{Z}$. Such a generator is usually known as the fundamental class of the pair $(M, \partial M)$. The inclusion $\mathbb{Z} \hookrightarrow \mathbb{R}$ induces a $\operatorname{map} l: H_{n}(M, \partial M ; \mathbb{Z}) \hookrightarrow H_{n}(M, \partial M ; \mathbb{R})=H_{n}(M, \partial M) \cong \mathbb{R}$, and we set $[M]_{\mathbb{R}}=$ $l\left([M]_{\mathbb{Z}}\right)$. The following definition is due to Gromov [7] (see also Thurston [23]):

Definition 1.1 The simplicial volume of $M$ is $\|M\|=\left\|[M]_{\mathbb{R}}\right\|$.

Since continuous maps induce norm nonincreasing maps on singular chains and a homotopy equivalence of pairs $f:(M, \partial M) \rightarrow(N, \partial N)$ between $n$-manifolds maps the fundamental class of $M$ into the fundamental class of $N$, it is readily seen that the simplicial volume of a compact manifold $M$ is a homotopy invariant of the pair $(M, \partial M)$.

As Gromov pointed out in his seminal work [7], even if it depends only on the homotopy type of a manifold, the simplicial volume is deeply related to the geometric structures that a manifold can carry. For example, closed manifolds which support negatively curved Riemannian metrics have nonvanishing simplicial volume, while the simplicial volume of flat or spherical manifolds is null (see eg Gromov [7]). Even if several vanishing and nonvanishing results for the simplicial volume have been established (see eg Gromov [7], Lafont and Schmidt [17] and Bucher-Karlsson [3]), it is maybe worth mentioning that, as far as the authors know, the exact value of nonnull simplicial volumes is known at the moment only in the following cases: a celebrated result by Gromov and Thurston (see Theorem 1.2 below) computes the simplicial volume of closed (and cusped) hyperbolic manifolds, and the simplicial volume of the product of compact orientable surfaces has been recently determined by Bucher-Karlsson [4]. 


\subsection{The simplicial volume of hyperbolic manifolds}

Let $n \geq 3$. Throughout the whole paper, by hyperbolic $n$-manifold we will mean a complete finite-volume hyperbolic $n$-manifold $M$ with compact (possibly empty) geodesic boundary. We recall that if $M$ is cusped, ie if it is noncompact, then $M$ naturally compactifies to a manifold with boundary $\bar{M}$ obtained by adding to $M$ a finite number of boundary $(n-1)$-manifolds supporting a flat structure (see Section 2.1 below).

Let $v_{n}$ be the supremum of volumes of geodesic $n$-simplices in hyperbolic $n$-space $\mathbb{H}^{n}$. It is well-known (see Haagerup and Munkholm [9] and Peyerimhoff [21]) that $v_{n}$ is equal to the volume of the geodesic regular ideal $n$-simplex. The following result is due to Thurston [23] and Gromov [7] (detailed proofs can be found in Benedetti and Petronio [2] for the closed case, and in Francaviglia [5] and Kuessner [16] for the cusped case):

Theorem 1.2 (Gromov, Thurston) Suppose $M$ is a hyperbolic $n$-manifold without boundary. Then $\|\bar{M}\| \neq 0$ and

$$
\frac{\operatorname{Vol}(M)}{\|\bar{M}\|}=v_{n} .
$$

A different result holds for hyperbolic manifolds with nonempty geodesic boundary:

Theorem $1.3[12 ; 15]$ Let $M$ be a hyperbolic $n$-manifold with nonempty geodesic boundary. Then $\|\bar{M}\| \neq 0$ and

$$
\frac{\operatorname{Vol}(M)}{\|\bar{M}\|}<v_{n} .
$$

In this paper we show how to control the gap between $\operatorname{Vol}(M) /\|\bar{M}\|$ and $v_{n}$ in terms of the ratio between the $(n-1)$-dimensional volume of $\partial M$ and the $n$-dimensional volume of $M$. More precisely, in Section 4 we prove the following:

Theorem 1.4 Let $\eta>0$. Then there exists $k>0$ depending only on $\eta$ and $n$ such that the following result holds: if $M$ is a hyperbolic $n$-manifold with nonempty geodesic boundary such that

$$
\frac{\operatorname{Vol}(\partial M)}{\operatorname{Vol}(M)} \leq k
$$

then

$$
\frac{\operatorname{Vol}(M)}{\|\bar{M}\|} \geq v_{n}-\eta .
$$


It is not difficult to show that for every $n \geq 3$ there exist compact hyperbolic $n-$ manifolds with nonempty disconnected geodesic boundary (see for example Gromov and Pietetski-Shapiro [8, Example 2.8.C]). Let $M$ be one such manifold, choose one connected component $B_{0}$ of $\partial M$ and let $M^{\prime}$ be the manifold obtained by mirroring $M$ along $\partial M \backslash B_{0}$, so $\partial M^{\prime}$ is isometric to two copies of $B_{0}$. For $i \geq 1$, we inductively construct $M_{i}$ by setting $M_{1}=M^{\prime}$ and defining $M_{i+1}$ as the manifold obtained by isometrically gluing one component of $\partial M_{i}$ to one component of $\partial M_{1}$. It is readily seen that $M_{i}$ is a compact hyperbolic $n$-manifold with nonempty geodesic boundary such that $\operatorname{Vol}\left(M_{i}\right)=2 i \operatorname{Vol}(M)$ and $\operatorname{Vol}\left(\partial M_{i}\right)=2 \operatorname{Vol}\left(B_{0}\right)$. We have therefore $\lim _{i \rightarrow \infty} \operatorname{Vol}\left(\partial M_{i}\right) / \operatorname{Vol}\left(M_{i}\right)=0$. Together with our main theorem, this easily implies the following:

Corollary 1.5 For every $\eta>0$, a compact hyperbolic $n$-manifold $M$ with nonempty geodesic boundary exists such that

$$
v_{n}>\frac{\operatorname{Vol}(M)}{\|M\|} \geq v_{n}-\eta .
$$

Finally, it is maybe worth mentioning that our proof of Theorem 1.4 can be applied word by word (with obvious simplifications) to hyperbolic manifolds without boundary, thus providing a somewhat new proof of Theorem 1.2 in the case of noncompact manifolds.

\subsection{Strategy of the proof}

Let $M$ be a hyperbolic $n$-manifold with possibly empty geodesic boundary. Once a straightening procedure is defined which allows us to compute the simplicial volume of $M$ only considering linear combinations of geodesic simplices, the inequality $\operatorname{Vol}(M) /\|\bar{M}\| \leq v_{n}$ is easily established. In the case without boundary, in order to prove the converse inequality (which fails in the case with boundary), one has to show that for every $\varepsilon>0$ a cycle $\alpha_{\varepsilon} \in C_{n}(\bar{M}, \partial \bar{M})$ exists which represents the fundamental class of $\bar{M}$ and is such that $\left\|\alpha_{\varepsilon}\right\| \leq \operatorname{Vol}(M) / v_{n}+\varepsilon$. Such a cycle is said to be $\varepsilon-$ efficient, and it is not difficult to show that a cycle is $\varepsilon$-efficient if its simplices all have hyperbolic volume close to $v_{n}$ (see eg Benedetti and Petronio [2]).

In the same spirit we prove here that if $M$ has nonempty geodesic boundary then the gap between $\operatorname{Vol}(M) /\|\bar{M}\|$ and $v_{n}$ is bounded from above by the amount of simplices in any fundamental cycle of $\bar{M}$ whose volume is forced to be far from $v_{n}$. In order to obtain Theorem 1.4 we then show how to control such amount of simplices in terms of the ratio between the $(n-1)$-dimensional volume of $\partial M$ and the $n$-dimensional volume of $M$. 
More precisely, in Section 2 we briefly describe some results about the geometry of hyperbolic manifolds with geodesic boundary. Section 3, which uses tools from Löh and Sauer [20, Section 4], is devoted to the description of a discrete version of Thurston's smearing construction which is very useful for exhibiting efficient cycles. Finally in Section 4 we provide the needed estimates on the norm of such cycles, thus concluding the proof of Theorem 1.4.

\section{Hyperbolic manifolds with geodesic boundary}

Let $n \geq 3$ and let $M$ be a hyperbolic $n$-manifold with nonempty geodesic boundary. This section is devoted to a brief description of the geometry of $M$.

\subsection{Natural compactification}

Since $\partial M$ is compact, $M$ decomposes as the union of a compact smooth manifold with boundary $N \subseteq M$ with $\partial M \subseteq N$ and a finite number of cusps of the form $T_{i} \times[0, \infty), i=1, \ldots, r$, where $T_{i}$ is a closed Euclidean $(n-1)$-manifold for every $i$ (see eg Kojima [13; 14] and Frigerio [6], where also the noncompact boundary case is considered). Moreover, $N$ can be chosen in such a way that each cusp $T_{i} \times[0, \infty)$ is isometric to the quotient of a closed horoball in $\mathbb{H}^{n}$ by a parabolic group of isometries.

Up to choosing "deeper" cusps, given $\varepsilon>0$ we may suppose that the volume of $M \backslash N$ is at most $\varepsilon$, and if this is the case we denote $N$ by the symbol $M_{\varepsilon}$. We also denote by $\partial M_{\varepsilon}$ the boundary of $M_{\varepsilon}$ as a topological manifold and we set $\operatorname{int}\left(M_{\varepsilon}\right)=M_{\varepsilon} \backslash \partial M_{\varepsilon}$. Observe that $\partial M_{\varepsilon}$ is given by the union of $\partial M$ and the boundaries of the deleted cusps.

The description of $M$ just given implies that there exists a well-defined piecewise smooth nearest point retraction $M \rightarrow M_{\varepsilon}$ which maps $M \backslash \operatorname{int}\left(M_{\varepsilon}\right)$ onto $\partial M_{\varepsilon}$. Moreover, $M$ admits a natural compactification $\bar{M}$ which is obtained by adding a closed Euclidean $(n-1)$-manifold for each cusp and is homeomorphic to $M_{\mathcal{E}}$.

\subsection{Universal covering}

Let $\pi: \widetilde{M} \rightarrow M$ be the universal covering of $M$. By developing $\widetilde{M}$ in $\mathbb{H}^{n}$ we can identify $\widetilde{M}$ with a convex polyhedron of $\mathbb{H}^{n}$ bounded by a countable number of disjoint geodesic hyperplanes. The group of the automorphisms of the covering $\pi: \widetilde{M} \rightarrow M$ can be identified in a natural way with a discrete torsion-free subgroup $\Gamma$ of $\operatorname{Isom}^{+}(\widetilde{M})<\operatorname{Isom}^{+}\left(\mathbb{H}^{n}\right)$ such that $M \cong \widetilde{M} / \Gamma$. Also recall that there exists an isomorphism $\pi_{1}(M) \cong \Gamma$, which is canonical up to conjugacy. With a slight abuse, from now on we refer to $\Gamma$ as to the fundamental group of $M$. 
The covering $\pi: \widetilde{M} \rightarrow M$ extends to a covering $\mathbb{H}^{n} \rightarrow \mathbb{H}^{n} / \Gamma=\widehat{M}$, which will still be denoted by $\pi$. Being the quotient of $\mathbb{H}^{n}$ by a discrete torsion-free group of isometries, $\widehat{M}$ is a complete (infinite-volume) hyperbolic manifold without boundary. The inclusion $\widetilde{M} \hookrightarrow \mathbb{H}^{n}$ induces an isometric inclusion $M \hookrightarrow \widehat{M}$ which realizes $M$ as the convex core of $\widehat{M}$ (see eg [6]). Therefore, there exists a well-defined piecewise smooth nearest point retraction of $\widehat{M}$ onto $M$, which maps $\widehat{M} \backslash \operatorname{int}(M)$ onto $\partial M$.

Let $\operatorname{ext}\left(M_{\varepsilon}\right)=\widehat{M} \backslash \operatorname{int}\left(M_{\varepsilon}\right)$. If $\varepsilon$ is sufficiently small, by composing the retractions $\widehat{M} \rightarrow M, M \rightarrow M_{\varepsilon}$ mentioned above we get a retraction $p: \widehat{M} \rightarrow M_{\varepsilon}$ such that $p\left(\operatorname{ext}\left(M_{\varepsilon}\right)\right) \subseteq \partial M_{\varepsilon}$. Such a map is piecewise smooth and induces a homotopy equivalence of pairs $p:\left(\widehat{M}, \operatorname{ext}\left(M_{\varepsilon}\right)\right) \rightarrow\left(M_{\varepsilon}, \partial M_{\varepsilon}\right)$.

Since $M$ retracts to $M_{\varepsilon}$ via a homotopy equivalence, the set $\widetilde{M}_{\varepsilon}=\pi^{-1}\left(M_{\varepsilon}\right) \subseteq \widetilde{M}$ is simply connected, and provides therefore the Riemannian universal covering of $M_{\varepsilon}$. If $\operatorname{ext}\left(\widetilde{M}_{\varepsilon}\right)=\mathbb{H}^{n} \backslash \operatorname{int}\left(\widetilde{M}_{\varepsilon}\right)$, then by construction $\operatorname{ext}\left(\widetilde{M}_{\varepsilon}\right)$ is a $\Gamma$-invariant disjoint union of closed half-spaces and closed horoballs. In particular, every component of $\operatorname{ext}\left(\widetilde{M}_{\varepsilon}\right)$ is convex.

\section{Straightening and smearing}

Introduced by Thurston in [23], the smearing construction plays a fundamental role in several proofs of Theorem 1.2 (see eg Thurston [23] and Kuessner [16]). Such a construction takes usually place in the setting of the so-called measure homology (see Thurston [23], Zastrow [25], Hansen [10] and Löh [19]). In order to make the proof of Theorem 1.4 as self-contained as possible and to get rid of some technicalities, we follow here some ideas described in Löh and Sauer [20], where a "discrete version" of the smearing construction is introduced.

\subsection{Straight simplices}

We now fix some notation we will be extensively using from now on. For $i \in \mathbb{N}$ we denote by $e_{i}$ the point $(0,0, \ldots, 1, \ldots, 0,0, \ldots) \in \mathbb{R}^{\mathbb{N}}$, where the unique nonzero coefficient is at the $i$-th entry (entries are indexed by $\mathbb{N}$, so $\left.(1,0, \ldots)=e_{0}\right)$. We denote by $\Delta_{p}$ the standard $p$-simplex, ie the convex hull of $e_{0}, \ldots, e_{p}$, and we observe that with this notation we have $\Delta_{p} \subseteq \Delta_{p+1}$. If $\sigma \in S_{p}(X)$ is a singular simplex, we let $\partial_{i} \sigma \in S_{p-1}(X)$ be the $i$-th face of $\sigma$.

Let $k \in \mathbb{N}$, and let $x_{0}, \ldots, x_{k}$ be points in $\mathbb{H}^{n}$. We recall here the well-known definition of straight simplex $\left[x_{0}, \ldots, x_{k}\right] \in S_{k}\left(\mathbb{H}^{n}\right)$ with vertices $x_{0}, \ldots, x_{k}$ : if $k=0$, then $\left[x_{0}\right]$ is the 0 -simplex with image $x_{0}$; if straight simplices have been defined for every 
$h \leq k$, then $\left[x_{0}, \ldots, x_{k+1}\right]: \Delta_{k+1} \rightarrow \mathbb{H}^{n}$ is determined by the following condition: for every $z \in \Delta_{k} \subseteq \Delta_{k+1}$, the restriction of $\left[x_{0}, \ldots, x_{k+1}\right]$ to the segment with endpoints $z, e_{k+1}$ is a constant speed parameterization of the geodesic joining $\left[x_{0}, \ldots, x_{k}\right](z)$ to $x_{k+1}$ (the fact that $\left[x_{0}, \ldots, x_{k+1}\right]$ is well-defined and continuous is an obvious consequence of the fact that any two given points in $\mathbb{H}^{n}$ are joined by a unique geodesic, and hyperbolic geodesics continuously depend on their endpoints - see also Remark 3.1 below). It is not difficult to show that the image of a straight $k$-simplex coincides with the hyperbolic convex hull of its vertices, and is therefore a (possibly degenerate) geodesic $k$-simplex. For later purposes we point out the following:

Remark 3.1 Since $\mathbb{H}^{n}$ is nonpositively curved, for every $p \in \mathbb{H}^{n}$ the exponential map $\exp _{p}: T_{p} \mathbb{H}^{n} \rightarrow \mathbb{H}^{n}$ is a diffeomorphism. Moreover, if $z \in \Delta_{k-1}$ and $q=$ $t z+(1-t) e_{k} \in \Delta_{k}$, then for every $(k+1)$-uple of vertices $\left(x_{0}, \ldots, x_{k}\right) \in\left(\mathbb{H}^{n}\right)^{k+1}$ we have

$$
\left[x_{0}, \ldots, x_{k}\right](q)=\exp _{x_{k}}\left(t \cdot \exp _{x_{k}}^{-1}\left(\left[x_{0}, \ldots, x_{k-1}\right](z)\right)\right) .
$$

Using this formula and an obvious inductive argument, it is not difficult to show that every straight simplex in $\mathbb{H}^{n}$ is smooth. Moreover, if we endow the space of straight simplices with the $C^{1}$-topology, then the map that takes every $(k+1)$-uple $\left(x_{0}, \ldots, x_{k}\right) \in\left(\mathbb{H}^{n}\right)^{k+1}$ into the straight simplex $\left[x_{0}, \ldots, x_{k}\right]$ is continuous.

Keeping notation from Sections 2.1 and 2.2, if $\widehat{M}$ is a hyperbolic $n$-manifold with universal covering $\pi: \mathbb{H}^{n} \rightarrow \widehat{M}$ then we say that $\sigma: \Delta_{k} \rightarrow \widehat{M}$ is straight if it is obtained by composing a straight simplex in $\mathbb{H}^{n}$ with the covering projection $\pi$.

The results stated in the following remarks will not be used in this paper.

Remark 3.2 Let $\sigma \in S_{k}(\widehat{M})$, take a lift $\widetilde{\sigma} \in S_{k}\left(\mathbb{H}^{n}\right)$ and define $\operatorname{str}_{k}(\sigma)=\pi \circ$ $\left[\widetilde{\sigma}\left(e_{0}\right), \ldots, \widetilde{\sigma}\left(e_{k}\right)\right]$. Since isometries preserve geodesics we have $\gamma \circ\left[x_{0}, \ldots, x_{k}\right]=$ $\left[\gamma\left(x_{0}\right), \ldots, \gamma\left(x_{k}\right)\right]$ for every $\gamma \in \Gamma$, so $\operatorname{str}_{k}(\sigma)$ does not depend on the choice of $\tilde{\sigma}$. It is well-known that $\operatorname{str}_{*}$ linearly extends to a chain endomorphism of $C_{*}(\widehat{M})$ which is algebraically homotopic to the identity.

Remark 3.3 Straight simplices may be defined in the much more general setting of nonpositively curved complete Riemannian manifolds. Almost all the properties of straight simplices (and of the associated straightening procedure) described above also hold in this wider context. It is maybe worth mentioning, however, that in the simply connected nonconstant curvature case, straight simplices need not be convex. 


\subsection{Some estimates on the volume of straight simplices}

As explained in Section 1.3, in order to prove our main theorem we have to construct fundamental cycles whose simplices have large volume. We are therefore interested in giving lower bounds on the volume of simplices which approximate, in a suitable sense, the geodesic ideal regular $n$-simplex.

For every $L>0$, let $q_{0}^{L}, \ldots q_{n}^{L} \in \mathbb{H}^{n}$ be the vertices of a fixed regular simplex with edgelength $L$ such that the embedding $\tau^{L}=\left[q_{0}^{L}, \ldots, q_{n}^{L}\right]$ of the standard simplex in $\mathbb{H}^{n}$ is orientation-preserving. For later purposes we insist that, as $L$ tends to infinity, the vertices of $\tau^{L}$ converge to the ideal vertices of a geodesic ideal regular simplex:

Lemma 3.4 We can choose the simplices $\tau^{L}, L>0$ in such a way that for every $i=0, \ldots, n$ we have $\lim _{L \rightarrow \infty} q_{i}^{L}=q_{i}^{\infty} \in \partial \mathbb{H}^{n}$, where $q_{0}^{\infty}, \ldots, q_{n}^{\infty}$ are the ideal vertices of a geodesic ideal regular positively-oriented $n$-simplex.

Proof Via an orientation-preserving identification of $\mathbb{H}^{n}$ with the Poincaré disk model of hyperbolic space, the ideal boundary $\partial \mathbb{H}^{n}$ is canonically identified with the unit sphere $S^{n-1}$, and if $q_{0}^{\infty}, \ldots, q_{n}^{\infty} \in S^{n-1}$ are the vertices of a positively-oriented regular Euclidean $n$-simplex inscribed in $S^{n-1}$, then the hyperbolic convex hull of the $q_{i}^{\infty}$ 's is in fact a regular ideal positively-oriented geodesic simplex with vertices $q_{0}^{\infty}, \ldots, q_{n}^{\infty}$. Let $\alpha_{i}$ be the geodesic ray which starts at the origin $O$ of the Poincaré disk model and is asymptotic to $q_{i}^{\infty}, i=0, \ldots, n$. For every $r>0$ let $p_{i}^{r}$ be the point on $\alpha_{i}$ at distance $r$ from $O$. It is easily seen that the straight simplex $\left[p_{0}^{r}, \ldots, p_{n}^{r}\right]$ is a regular simplex of edgelength $\ell(r)$, where $\ell:(0, \infty) \rightarrow(0, \infty)$ is increasing and bijective. In order to conclude it is now sufficient to set $q_{i}^{L}=p_{i}^{\ell^{-1}}(L)$.

We are now interested in studying limits, as $L$ tends to infinity, of volumes of straight simplices which approximate $\tau^{L}$.

Definition 3.5 We denote by $R_{L}$ be the set of all straight $n$-simplices $\sigma$ in $\mathbb{H}^{n}$ satisfying the following property: there exists $g \in \operatorname{Isom}^{+}\left(\mathbb{H}^{n}\right)$ such that the distance between $g\left(q_{i}^{L}\right)$ and the $i$-th vertex of $\sigma$ is at most one for every $i=0, \ldots, n$. Moreover, we set

$$
V_{L}=\inf \left\{\operatorname{Vol}_{\mathrm{alg}}(\sigma) \mid \sigma \in R_{L}\right\},
$$

where $\mathrm{Vol}_{\mathrm{alg}}$ is the signed volume of $\sigma$, ie the value obtained by integrating the volume form of $\mathbb{H}^{n}$ on $\sigma$ (so $\left|\operatorname{Vol}_{\text {alg }}(\sigma)\right|=\operatorname{Vol}(\operatorname{Im}(\sigma))$ ).

As pointed out in Remark 3.1, straight simplices continuously depend (with respect to the $C^{1}$-topology) on their vertices, and this readily implies that the algebraic volume 
of a straight simplex continuously depends on its vertices. Together with a similar (but deeper) continuity result for possibly ideal geodesic simplices (see Ratcliffe [22, Theorem 11.3.2]), this fact allows us to prove the following:

\section{Lemma 3.6 We have}

$$
\lim _{L \rightarrow \infty} V_{L}=v_{n}
$$

In particular, there exists $L_{0}>0$ such that if $L \geq L_{0}$ then every element of $R_{L}$ is an embedded positively-oriented straight simplex in $\mathbb{H}^{n}$.

Proof Observe that by the very definitions a hyperbolic straight simplex is embedded and positively-oriented if and only if it has positive algebraic volume. Therefore it is sufficient to show that if $\left\{L_{j}\right\}_{j \in \mathbb{N}}$ is a sequence of positive real numbers such that $\lim _{j} L_{j}=+\infty$ and $\sigma^{j} \in R_{L_{j}}$, then $\lim _{j} \operatorname{Vol}_{\mathrm{alg}}\left(\sigma^{j}\right)=v_{n}$ (here and in what follows, whenever $\{f(j)\}_{j \in \mathbb{N}}$ is a real sequence we denote simply by $\lim _{j} f(j)$ the limit $\lim _{j \rightarrow \infty} f(j)$, if it exists).

Recall from Lemma 3.4 that the simplices $\tau^{L}, L>0$ have been chosen in such a way that $\lim _{L \rightarrow \infty} q_{i}^{L}=q_{i}^{\infty} \in \partial \mathbb{H}^{n}$ for $i=0, \ldots, n$, where $q_{0}^{\infty}, \ldots, q_{n}^{\infty}$ are the ideal vertices of a regular ideal positively-oriented geodesic $n$-simplex $\tau^{\infty}$.

For every $i=0, \ldots, n$, let $w_{i}^{j}$ be the $i$-th vertex of $\sigma^{j} \in R_{L_{j}}$. Then by the very definitions there exists $g^{j} \in \operatorname{Isom}^{+}\left(\mathbb{H}^{n}\right)$ such that for every $i=0, \ldots, n$ we have $d\left(g^{j}\left(w_{i}^{j}\right), q_{i}^{L_{j}}\right) \leq 1$, and this readily implies $\lim _{j} g^{j}\left(w_{i}^{j}\right)=q_{i}^{\infty}$. As a consequence we get

$$
\lim _{j} \operatorname{Vol}\left(\sigma^{j}\right)=\lim _{j} \operatorname{Vol}\left(g^{j} \cdot \sigma^{j}\right)=\operatorname{Vol}\left(\tau^{\infty}\right)=v_{n},
$$

where the first equality is due to the fact that isometries preserve the hyperbolic volume, while the second one is due to the fact that the hyperbolic volume of a geodesic simplex with vertices in $\mathbb{H}^{n} \cup \partial \mathbb{H}^{n}$ is a continuous function of its vertices (see Ratcliffe [22, Theorem 11.3.2]).

Suppose now by contradiction that for every $j_{0} \in \mathbb{N}$ there exists $j \geq j_{0}$ such that $\sigma^{j}$ is not positively-oriented. Since the $g^{j}$ 's are orientation-preserving, up to passing to a subsequence we may then suppose $\operatorname{Vol}_{\text {alg }}\left(g^{j} \cdot \sigma^{j}\right)=\operatorname{Vol}_{\text {alg }}\left(\sigma^{j}\right) \leq 0$ for every $j \in \mathbb{N}$. For every $j \in \mathbb{N}, i=0, \ldots, n$ let $v_{i}^{j}(t), t \in[0,1]$ be a geodesic path joining $g^{j}\left(w_{i}^{j}\right)$ with $q_{i}^{L_{j}}$. Since $\tau^{L_{j}}$ is positively-oriented we have $\operatorname{Vol}_{\text {alg }}\left(\tau^{L_{j}}\right)>0$ for every $j \in \mathbb{N}$. Moreover, as observed above the algebraic volume of a straight simplex continuously depends on its vertices, so for every $j \geq j_{0}$ there exists $t_{j} \in[0,1]$ such that the straight simplex $\hat{\sigma}^{j}$ with vertices $v_{0}^{j}\left(t_{j}\right), \ldots, v_{n}^{j}\left(t_{j}\right)$ has null algebraic volume, whence in particular $\lim _{j} \operatorname{Vol}\left(\hat{\sigma}^{j}\right)=\lim _{j}\left|\operatorname{Vol}_{\mathrm{alg}}\left(\hat{\sigma}^{j}\right)\right|=0$. However, we have by construction 
$\hat{\sigma}^{j} \in R_{L_{j}}$, so, by applying to the $\hat{\sigma}^{j}$ 's the argument described in the first part of the proof, we get $\lim _{j} \operatorname{Vol}\left(\hat{\sigma}^{j}\right)=v_{n}$. This contradiction shows that there exists $j_{0}$ such that $\operatorname{Vol}_{\text {alg }}\left(\sigma^{j}\right) \geq 0$ for every $j \geq j_{0}$.

Together with Equation (1), this implies $\lim _{j} \operatorname{Vol}_{\mathrm{alg}}\left(\sigma^{j}\right)=\lim _{j} \operatorname{Vol}\left(\sigma^{j}\right)=v_{n}$, whence the conclusion.

\subsection{Haar measure}

Let $G$ be a locally compact Hausdorff topological group and $\mathcal{A}$ be the $\sigma$-algebra of the Borel subsets of $G$. A measure $\mu$ on $\mathcal{A}$ is called regular if for each $A \in \mathcal{A}$

$$
\begin{aligned}
& \mu(A)=\sup \{\mu(K) \mid K \text { compact set, } K \subseteq A\}, \\
& \mu(A)=\inf \{\mu(U) \mid U \text { open set, } A \subseteq U\} .
\end{aligned}
$$

Definition 3.7 A Haar measure $\mu_{G}$ on $G$ is a nonnegative regular measure $\mu_{G}$ on the $\sigma$-algebra $\mathcal{A}$ such that

- $\mu_{G}(K)<\infty$ for each compact set $K \in \mathcal{A}$;

- $\mu_{G}(A) \neq 0$ for each nonempty open set $A \in \mathcal{A}$;

- $\mu_{G}(g A)=\mu_{G}(A)$ for all $g \in G$ and for all $A \in \mathcal{A}$, namely the measure is left-invariant.

It is well-known that every locally compact Hausdorff group admits a Haar measure, which is unique up to multiplication by a positive constant $[11 ; 24]$. The group $G$ is called unimodular if each left-invariant Haar measure on it is also right-invariant.

From now on we denote by $G$ the group $\operatorname{Isom}\left(\mathbb{H}^{n}\right)$ of isometries of $\mathbb{H}^{n}$, endowed with the compact-open topology, and by $G^{+} \subseteq G$ (resp. $G^{-} \subseteq G$ ) the subset of orientation-preserving (resp. orientation-reversing) elements of $G$. Of course, $G^{+}$is a subgroup of $G$, and it is well-known that $G^{+}, G^{-}$are the connected components of $G$. Also observe that since $M$ is orientable we have $\Gamma<G^{+}$.

Proposition 3.8 [2; 22] The group $G$ is locally compact and unimodular. Moreover, the Haar measure $\mu_{G}$ on $G$ can be normalized in such a way that the following condition holds: for every basepoint $x_{0} \in \mathbb{H}^{n}$ and every Borel set $R \subseteq \mathbb{H}^{n}$, the Haar measure $\mu_{G}\left(G_{R}\right)$ of the Borel set

$$
G_{R}=\left\{g \in G \mid g\left(x_{0}\right) \in R\right\}
$$

is equal to the hyperbolic volume of $R$. 
From now on we fix a Haar measure $\mu_{G}$ on $G$ satisfying the normalization condition described in Proposition 3.8. Keeping notation from the preceding section, $\Gamma$ acts properly discontinuously on $G$ via left translations as a group of measure-preserving diffeomorphisms, so if $W$ is a Borel subset of $\Gamma \backslash G$ we can set

$$
\mu_{\Gamma \backslash G}(W)=\mu_{G}(\widetilde{W}),
$$

where $\widetilde{W} \subseteq G$ is any Borel set that projects bijectively onto $W$. It is readily seen that this definition of $\mu_{\Gamma \backslash G}(W)$ does not depend on the choice of $\widetilde{W}$, and that $\mu_{\Gamma \backslash G}$ is in fact a regular right-invariant measure on $\Gamma \backslash G$. The following lemma will prove useful later:

Lemma 3.9 Let $A \subseteq \widehat{M}$ be a Borel subset, fix a basepoint $x_{0} \in \mathbb{H}^{n}$ and set

$$
T^{ \pm}=\left\{g \in G^{ \pm} \mid \pi\left(g\left(x_{0}\right)\right) \in A\right\} .
$$

Then $T^{ \pm}$is a Borel subset of $G$ such that $\gamma \cdot T^{ \pm}=T^{ \pm}$for every $\gamma \in \Gamma$ and $\mu_{\Gamma \backslash G}\left(\Gamma \backslash T^{ \pm}\right)=\operatorname{Vol}(A) / 2$, where Vol denotes the measure induced by the hyperbolic volume form of $\widehat{M}$.

Proof Let us consider the map $f_{x_{0}}^{ \pm}: G^{ \pm} \rightarrow \widehat{M}$ defined by $f_{x_{0}}^{ \pm}(g)=\pi\left(g\left(x_{0}\right)\right)$. Then $f_{x_{0}}^{ \pm}$is continuous, and for every $\gamma \in \Gamma$ we have $f_{x_{0}}^{ \pm}(g)=f_{x_{0}}^{ \pm}(\gamma g)$. Being the preimage of $A$ under $f_{x_{0}}^{ \pm}$, the set $T^{ \pm}$is therefore Borel and invariant with respect to the left action of $\Gamma$ on $G^{ \pm}$. Moreover, if $D \subseteq \mathbb{H}^{n}$ is a Borel set of representatives for the action of $\Gamma$ on $\mathbb{H}^{n}$, then the set $\widehat{T}^{ \pm}=\left\{g \in G^{ \pm} \mid g\left(x_{0}\right) \in D \cap \pi^{-1}(A)\right\}$ is a Borel set of representatives for the left action of $\Gamma$ on $T^{ \pm}$. We have therefore $\mu_{\Gamma \backslash G}\left(\Gamma \backslash T^{ \pm}\right)=$ $\mu_{G}\left(\hat{T}^{ \pm}\right)$, so in order to conclude it is sufficient to show that $\mu_{G}\left(\widehat{T}^{+}\right)=\mu_{G}\left(\hat{T}^{-}\right)$and $\mu_{G}\left(\widehat{T}^{+}\right)+\mu_{G}\left(\widehat{T}^{-}\right)=\operatorname{Vol}(A)$.

Since $\widehat{T}^{-}=\widehat{T}^{+} \cdot g$, where $g \in G^{-}$is any orientation-reversing isometry fixing $x_{0}$, the first equality is an immediate consequence of the right-invariance of $\mu_{G}$. On the other hand the chosen normalization of $\mu_{G}$ readily gives $\operatorname{Vol}\left(D \cap \pi^{-1}(A)\right)=$ $\mu_{G}\left(\widehat{T}^{+} \cup \widehat{T}^{-}\right)=\mu_{G}\left(\widehat{T}^{+}\right)+\mu_{G}\left(\widehat{T}^{-}\right)$, where the last equality is due to the fact that $\widehat{T}^{+} \cap \widehat{T}^{-} \subseteq G^{+} \cap G^{-}=\varnothing$. Since the projection $\left.\pi\right|_{D}: D \rightarrow \widehat{M}$ is measure-preserving, this implies in turn $\operatorname{Vol}(A)=\operatorname{Vol}\left(D \cap \pi^{-1}(A)\right)=\mu_{G}\left(\widehat{T}^{+}\right)+\mu_{G}\left(\widehat{T}^{-}\right)$, whence the conclusion.

\section{$3.4 \Gamma$-Nets}

In order to define a "discrete" smearing procedure in the spirit of [20] we now need to introduce the notion of $\Gamma$-net. A $\Gamma-n e t$ in $\mathbb{H}^{n}$ is given by a discrete subset $\widetilde{\Lambda} \subseteq \mathbb{H}^{n}$ (called set of vertices) and a collection of Borel sets $\left\{\widetilde{B}_{x}\right\}_{x \in \tilde{\Lambda}}$ (called cells) such that the following conditions hold: 
(1) $x \in \widetilde{B}_{x}$ for every $x \in \tilde{\Lambda}, \mathbb{H}^{n}=\bigcup_{x \in \widetilde{\Lambda}} \widetilde{B}_{x}$ and $\widetilde{B}_{x} \cap \widetilde{B}_{y}=\varnothing$ for every $x, y \in \widetilde{\Lambda}$ with $x \neq y$.

(2) $\gamma(\tilde{\Lambda})=\tilde{\Lambda}$ for every $\gamma \in \Gamma$ and $\gamma\left(\widetilde{B}_{x}\right)=\widetilde{B}_{\gamma(x)}$ for every $x \in \tilde{\Lambda}, \gamma \in \Gamma$.

(3) $\operatorname{diam}\left(\widetilde{B}_{x}\right) \leq 1$ for every $x \in \tilde{\Lambda}$.

(4) If $\widetilde{K}$ is a connected component of $\operatorname{ext}\left(\widetilde{M}_{\varepsilon}\right)$, then $\widetilde{K}=\bigcup_{x \in \tilde{\Lambda} \cap \widetilde{K}} \widetilde{B}_{x}$.

We begin with the following:

Lemma 3.10 There exists a $\Gamma$-net.

Proof Let $\left\{T_{i}\right\}_{i \in \mathbb{N}}$ be a smooth triangulation of $\widehat{M}$ which restricts to a smooth triangulation of $M_{\varepsilon}$ and is such that $\operatorname{diam}\left(T_{i}\right) \leq 1 / 2$ for every $i \in \mathbb{N}$. Since $M_{\varepsilon}$ is compact, the set of simplices of the triangulation whose internal part is contained in $M_{\varepsilon}$ is finite, so we may assume that indices are ordered in such a way that $i<j$ for every pair of simplices such that $\operatorname{int}\left(T_{i}\right) \subseteq M_{\varepsilon}$ and $\operatorname{int}\left(T_{j}\right) \nsubseteq M_{\varepsilon}$.

If $x_{i}$ is any point in $\operatorname{int}\left(T_{i}\right)$ we now set $\Lambda=\left\{x_{i}\right\}_{i \in \mathbb{N}}$ and $B_{x_{i}}=T_{i} \backslash\left(\bigcup_{j>i} T_{j}\right)$. By construction $B_{x_{i}}$ is a simply connected Borel subset of $\widehat{M}$ for every $i \in \mathbb{N}$, and if $K$ is a connected component of $\operatorname{ext}\left(M_{\varepsilon}\right)$, then $K=\bigcup_{x \in \Lambda \cap K} B_{x}$.

Let now $\tilde{\Lambda}=\pi^{-1}(\Lambda)$. For $i \in \mathbb{N}$, since $B_{x_{i}}$ is simply connected, every point $\tilde{x} \in \pi^{-1}\left(x_{i}\right)$ is contained in exactly one connected component $\widetilde{B}_{\widetilde{x}}$ of $\pi^{-1}\left(B_{x_{i}}\right) \subseteq \mathbb{H}^{n}$. It is now readily seen that the pair $\left(\widetilde{\Lambda},\left\{\widetilde{B}_{x}\right\}_{x \in \tilde{\Lambda}}\right)$ is a $\Gamma$-net.

\subsection{Smearing}

We now fix $L>0$, and for $i=0, \ldots, n$ we denote simply by $q_{i}$ the vertex $q_{i}^{L}$ of the straight simplex $\tau^{L}$ introduced in Section 3.2. We also fix a $\Gamma-$ net $\left(\tilde{\Lambda},\left\{\widetilde{B}_{x}\right\}_{x \in \tilde{\Lambda}}\right)$ and let $S_{n}^{\Lambda}(\widehat{M})$ denote the set of straight $n$-simplices in $\widehat{M}$ with vertices in $\Lambda=\pi(\widetilde{\Lambda})$. We would like to define real coefficients $a_{\sigma}$ in such a way that the sum

$$
\sum_{\sigma \in S_{n}^{\Lambda}(\widehat{M})} a_{\sigma} \sigma
$$

is finite and defines a cycle in $C_{n}\left(\widehat{M}, \operatorname{ext}\left(M_{\varepsilon}\right)\right)$. Roughly speaking, the coefficient $a_{\sigma}$ will measure the difference between the accuracy with which $\sigma$ approximates a positively-oriented isometric copy of $\tau^{L}$ and the accuracy with which it approximates a negatively-oriented isometric copy of $\tau^{L}$. 
So let us fix $\sigma \in S_{n}^{\Lambda}(\widehat{M})$, let $\widetilde{\sigma}$ be a lift of $\sigma$ to $\mathbb{H}^{n}$, and let $\tilde{x}_{0}, \ldots, \tilde{x}_{n}$ be the vertices of $\widetilde{\sigma}$. Since $\sigma \in S_{n}^{\Lambda}$ we have $\tilde{x}_{j} \in \widetilde{\Lambda}$ for every $j$, and we denote by $\widetilde{B}_{j}$ the cell of the net containing $\tilde{x}_{j}$. It is readily seen that the sets

$$
\begin{aligned}
& \Omega_{\tilde{\sigma}}^{ \pm}=\left\{g \in G^{ \pm} \mid g\left(q_{j}\right) \in \widetilde{B}_{j} \text { for every } j\right\}, \\
& \widetilde{\Omega}_{\sigma}^{ \pm}=\bigcup_{\gamma \in \Gamma} \Omega_{\gamma \cdot \widetilde{\sigma}}^{ \pm}
\end{aligned}
$$

are Borel. Since different lifts of $\sigma$ differ by the action of an element of $\Gamma$, the set $\widetilde{\Omega}_{\sigma}^{ \pm}$only depends on $\sigma$, ie it is independent of the chosen lift $\widetilde{\sigma}$. Moreover, as a consequence of property (2) of $\Gamma$-nets it is easy to see that for every $\gamma \in \Gamma$ we have $\gamma \cdot \Omega_{\tilde{\sigma}}^{ \pm}=\Omega_{\gamma \cdot \widetilde{\sigma}}^{ \pm}$, so

$$
\widetilde{\Omega}_{\sigma}^{ \pm}=\bigcup_{\gamma \in \Gamma} \gamma \cdot \Omega(\underset{\tilde{\sigma}}{ \pm}
$$

and in particular $\gamma \cdot \widetilde{\Omega}_{\sigma}^{ \pm}=\widetilde{\Omega}_{\sigma}^{ \pm}$for every $\gamma \in \Gamma$.

We now claim that $\Omega \underset{\tilde{\sigma}}{ \pm}$ provides a Borel set of representatives for the action of $\Gamma$ on $\widetilde{\Omega}_{\sigma}^{ \pm}$. In fact, let us suppose by contradiction that there exist $\gamma, \gamma^{\prime} \in \Gamma$ with $\gamma \neq \gamma^{\prime}$ and $\Omega_{\gamma \cdot \widetilde{\sigma}}^{ \pm} \cap \Omega_{\gamma^{\prime}}^{ \pm} \neq \varnothing$. If $g \in \Omega_{\gamma \cdot \widetilde{\sigma}}^{ \pm} \cap \Omega_{\gamma^{\prime}}^{ \pm}$, then $g\left(q_{0}\right) \in \gamma\left(\widetilde{B}_{0}\right) \cap \gamma^{\prime}\left(\widetilde{B}_{0}\right) \neq \varnothing$, and by property (2) of $\Gamma$-nets this readily implies $\gamma\left(\tilde{x}_{0}\right)=\gamma^{\prime}\left(\tilde{x}_{0}\right)$, whence $\gamma^{-1}\left(\gamma^{\prime}\left(\tilde{x}_{0}\right)\right)=\tilde{x}_{0}$, a contradiction since the action of $\Gamma$ on $\mathbb{H}^{n}$ is free.

We denote by $\Omega_{\sigma}^{ \pm} \subseteq \Gamma \backslash G$ the quotient set $\Omega_{\sigma}^{ \pm}=\Gamma \backslash \widetilde{\Omega}_{\sigma}^{ \pm}$, and we observe that as a consequence of the claim just proved we have $\mu_{\Gamma \backslash G}\left(\Omega_{\sigma}^{ \pm}\right)=\mu_{G}\left(\Omega_{\widetilde{\sigma}}^{ \pm}\right)$.

Remark 3.11 In principle, in what follows one could work only with the sets $\Omega \underset{\widetilde{\sigma}}{ \pm}$ and the Haar measure $\mu_{G}$ on $G$, rather than define also $\Omega_{\sigma}^{ \pm}$and the quotient measure $\mu_{\Gamma \backslash G}$ on $\Gamma \backslash G$. However, we have preferred to introduce some more notions (and notation) since this choice will allow us to simplify some proofs, exploiting the properties of the most convenient object as and when.

We now divide the simplices of $S_{n}^{\Lambda}(\widehat{M})$ into different classes. We denote by $W^{+}$ (resp. $W^{-}$) the set of simplices which intersect $\operatorname{int}\left(M_{\varepsilon}\right)$ and are "almost isometric" to a positively-oriented (resp. negatively-oriented) copy of $\tau^{L}$ :

$$
W^{ \pm}=\left\{\sigma \in S_{n}^{\Lambda}(\widehat{M}) \mid \operatorname{Im}(\sigma) \cap \operatorname{int}\left(M_{\varepsilon}\right) \neq \varnothing \text { and } \Omega_{\sigma}^{ \pm} \neq \varnothing\right\} .
$$

Moreover, we denote by $W_{\text {int }}^{ \pm}$the subset of $W^{ \pm}$given by those simplices whose image is entirely contained in $\operatorname{int}\left(M_{\varepsilon}\right)$ :

$$
W_{\text {int }}^{ \pm}=\left\{\sigma \in W^{ \pm} \mid \operatorname{Im}(\sigma) \subseteq \operatorname{int}\left(M_{\varepsilon}\right)\right\},
$$


and we finally define

$$
W=W^{+} \cup W^{-}, \quad W_{\text {int }}=W_{\text {int }}^{+} \cup W_{\text {int }}^{-}, \quad W_{\text {ext }}=W \backslash W_{\text {int }} .
$$

For every $\sigma \in W$ we now set

$$
b_{\sigma}^{ \pm}=\mu_{\Gamma \backslash G}\left(\Omega_{\sigma}^{ \pm}\right), \quad a_{\sigma}=b_{\sigma}^{+}-b_{\sigma}^{-} .
$$

We will prove soon that $W$ is finite and that each $a_{\sigma}$ is a well-defined real number (see Lemma 3.13). Moreover, we will show in Proposition 3.14 that the sum $\sum_{\sigma \in W} a_{\sigma} \sigma$ defines a relative cycle in $C_{n}\left(\widehat{M}, \operatorname{ext}\left(M_{\varepsilon}\right)\right)$, which defines in turn a cycle in $C_{n}\left(M_{\varepsilon}, \partial M_{\varepsilon}\right)$ via a projection which does not affect simplices supported in $\operatorname{int}\left(M_{\varepsilon}\right)$. Therefore if $L$ is large and "most" simplices of $W$ are contained in $\operatorname{int}\left(M_{\varepsilon}\right)$, then a fundamental cycle for $M_{\varepsilon}$ exists most simplices of which have volume close to $v_{n}$. As explained in Section 1.3, this is sufficient for proving that the simplicial volume of $M_{\varepsilon}$ (whence of $\bar{M}$ ) is close to $\operatorname{Vol}\left(M_{\varepsilon}\right) / v_{n}$ (whence to $\operatorname{Vol}(M) / v_{n}$ ).

Lemma 3.12 If $\tilde{\sigma} \in S_{n}\left(\mathbb{H}^{n}\right)$ is a lift of a simplex $\sigma \in W$, then $\operatorname{diam}(\operatorname{Im}(\widetilde{\sigma})) \leq L+2$.

Proof The edges of $\tau^{L}$ have length $L$, and if $\sigma \in W$ then the vertices of $\widetilde{\sigma}$ are at distance at most 1 from the vertices of an isometric copy of $\tau^{L}$. This implies that the distance between any two vertices of $\widetilde{\sigma}$ is at most $L+2$. But $\tilde{\sigma}$ is the convex hull of its vertices and the hyperbolic distance is convex, so the diameter of $\widetilde{\sigma}$ is realized by the distance between two vertices.

From now on, for $Y \subseteq \widehat{M}$ (resp. $Y \subseteq \mathbb{H}^{n}$ ) and $R \geq 0$ we denote by $N_{R}(Y)$ the closed $R$-neighbourhood of $Y$ in $\widehat{M}$ (resp. in $\mathbb{H}^{n}$ ).

Lemma 3.13 The set $W$ is finite and

$$
\begin{aligned}
& \operatorname{Vol}\left(M_{\varepsilon} \backslash N_{L+3}\left(\partial M_{\varepsilon}\right)\right) \leq 2 \sum_{\sigma \in W_{\text {int }}^{+}} b_{\sigma}^{+} \leq 2 \sum_{\sigma \in W^{+}} b_{\sigma}^{+} \leq \operatorname{Vol}\left(N_{L}\left(M_{\varepsilon}\right)\right), \\
& \operatorname{Vol}\left(M_{\varepsilon} \backslash N_{L+3}\left(\partial M_{\varepsilon}\right)\right) \leq 2 \sum_{\sigma \in W_{\mathrm{int}}^{-}} b_{\sigma}^{-} \leq 2 \sum_{\sigma \in W^{-}} b_{\sigma}^{-} \leq \operatorname{Vol}\left(N_{L}\left(M_{\varepsilon}\right)\right) .
\end{aligned}
$$

Proof Let $D \subseteq \mathbb{H}^{n}$ be a compact fundamental region for the action of $\Gamma$ on $\widetilde{M}_{\mathcal{\varepsilon}}$. If $\sigma \in W$ there exists a lift $\tilde{\sigma} \in S_{n}\left(\mathbb{H}^{n}\right)$ of $\sigma$ such that $\operatorname{Im}(\widetilde{\sigma}) \cap D \neq \varnothing$. By Lemma 3.12 the diameter of $\widetilde{\sigma}$ is at most $L+2$, so $\operatorname{Im}(\widetilde{\sigma}) \subseteq N_{L+2}(D)$. However, $\tilde{\Lambda}$ is discrete and $N_{L+2}(D)$ is compact, so the number of straight simplices in $\mathbb{H}^{n}$ which are contained in $N_{L+2}(D)$ and have vertices in $\widetilde{\Lambda}$ is finite. Since every $\sigma \in W$ is obtained by composing such a simplex with the covering projection $\pi$ we get that also $W$ is finite. 
We now prove the first sequence of inequalities of the statement, the proof of the second one being very similar. We define the subsets $H_{L}^{+}, K_{L}^{+} \subseteq G^{+}$as follows:

$$
\begin{aligned}
& K_{L}^{+}=\left\{g \in G^{+} \mid g\left(q_{0}\right) \in \widetilde{M}_{\varepsilon} \backslash N_{L+3}\left(\partial \widetilde{M}_{\varepsilon}\right)\right\}, \\
& H_{L}^{+}=\left\{g \in G^{+} \mid g\left(q_{0}\right) \in N_{L}\left(\widetilde{M}_{\varepsilon}\right)\right\} .
\end{aligned}
$$

By Lemma 3.9 these sets are $\Gamma$-invariant Borel subsets of $G^{+}$such that

$$
\mu_{\Gamma \backslash G}\left(\Gamma \backslash K_{L}^{+}\right)=\frac{\operatorname{Vol}\left(M_{\varepsilon} \backslash N_{L+3}\left(\partial M_{\varepsilon}\right)\right)}{2}, \quad \mu_{\Gamma \backslash G}\left(\Gamma \backslash H_{L}^{+}\right)=\frac{\operatorname{Vol}\left(N_{L}\left(M_{\varepsilon}\right)\right)}{2} .
$$

We now show that

$$
K_{L}^{+} \subseteq \bigcup_{\sigma \in W_{\text {int }}^{+}} \widetilde{\Omega}_{\sigma}^{+} \subseteq \bigcup_{\sigma \in W^{+}} \widetilde{\Omega}_{\sigma}^{+} \subseteq H_{L}^{+}
$$

and these inclusions readily imply that

$$
\Gamma \backslash K_{L}^{+} \subseteq \bigcup_{\sigma \in W_{\mathrm{int}}^{+}} \Omega_{\sigma}^{+} \subseteq \bigcup_{\sigma \in W^{+}} \Omega_{\sigma}^{+} \subseteq \Gamma \backslash H_{L}^{+} .
$$

Suppose first $g \in K_{L}^{+}$. Then $g\left(q_{0}\right)$ lies in $\widetilde{M}_{\varepsilon} \backslash N_{L+3}\left(\partial \widetilde{M}_{\varepsilon}\right)$, so if $y_{i} \in \tilde{\Lambda}$ is the vertex of the cell containing $g\left(q_{i}\right)$, then $y_{0}$ lies in $\widetilde{M}_{\varepsilon} \backslash N_{L+2}\left(\partial \widetilde{M}_{\varepsilon}\right)$. Let now $\widetilde{\sigma}$ be the straight simplex with vertices $y_{0}, \ldots, y_{n}$ and set $\sigma=\pi \circ \tilde{\sigma}$. By Lemma 3.12 the image of $\widetilde{\sigma}$ lies in $\operatorname{int}\left(\widetilde{M}_{\varepsilon}\right)$, so $\operatorname{Im}(\sigma) \subseteq \operatorname{int}\left(M_{\varepsilon}\right)$. Moreover, we have by construction $g \in \Omega_{\tilde{\sigma}}^{+} \subseteq \widetilde{\Omega}_{\sigma}^{+}$, so $\Omega_{\sigma}^{+}=\Gamma \backslash \widetilde{\Omega}_{\sigma}^{+} \neq \varnothing$ and $\sigma \in W_{\text {int }}^{+}$. We have thus proved the first inclusion in (3).

The second inclusion is an obvious consequence of the inclusion $W_{\text {int }}^{+} \subseteq W^{+}$, so we are left to prove the last inclusion in (3). Suppose $g \in \widetilde{\Omega}_{\sigma}^{+}$for some $\sigma \in W^{+}$. Then there exists a lift $\tilde{\sigma}$ of $\sigma$ with vertices $y_{0}, \ldots, y_{n} \in \widetilde{\Lambda}$ such that $g \in \Omega_{\tilde{\sigma}}^{+}$, whence $g\left(q_{i}\right) \in \widetilde{B}_{y_{i}}$ for every $i=0, \ldots, n$. Also observe that since $\sigma \in W^{+}$we have $\operatorname{Im}(\widetilde{\sigma}) \cap \operatorname{int}\left(\widetilde{M}_{\varepsilon}\right) \neq \varnothing$. Suppose by contradiction $g\left(q_{0}\right) \notin N_{L}\left(\widetilde{M}_{\varepsilon}\right)$. Having diameter $L$ and being connected, the image of $g \circ \tau^{L}$ is then contained in a component $\widetilde{K}$ of $\operatorname{ext}\left(\widetilde{M}_{\mathcal{E}}\right)$. Since $g\left(q_{i}\right) \in \widetilde{B}_{y_{i}}$, by property (4) in the definition of $\Gamma$-net we have therefore $y_{i} \in \widetilde{K}$ for every $i$, so $\operatorname{Im}(\widetilde{\sigma}) \subseteq \widetilde{K}$ by convexity of $\widetilde{K}$, whence $\operatorname{Im}(\widetilde{\sigma}) \cap \operatorname{int}\left(\widetilde{M}_{\varepsilon}\right)=\varnothing$, a contradiction. This concludes the proof of (3), whence of (4).

Since the $\Omega_{\sigma}^{+}$'s are mutually disjoint, condition (4) now implies

$$
\mu_{\Gamma \backslash G}\left(\Gamma \backslash K_{L}^{+}\right) \leq \sum_{\sigma \in W_{\text {int }}^{+}} \mu_{\Gamma \backslash G}\left(\Omega_{\sigma}^{+}\right) \leq \sum_{\sigma \in W^{+}} \mu_{\Gamma \backslash G}\left(\Omega_{\sigma}^{+}\right) \leq \mu_{\Gamma \backslash G}\left(\Gamma \backslash H_{L}^{+}\right) .
$$

By Equations (2) and the very definition of the $b_{\sigma}^{+}$'s, these inequalities are in fact equivalent to the first sequence of inequalities in the statement. 
We now set

$$
\zeta_{L, \varepsilon}=\sum_{\sigma \in W} a_{\sigma} \sigma \in C_{n}(\widehat{M})
$$

Proposition 3.14 We have $d \zeta_{L, \varepsilon} \in C_{n-1}\left(\operatorname{ext}\left(M_{\varepsilon}\right)\right)$, so $\zeta_{L, \varepsilon}$ is a relative $n$-cycle in $C_{n}\left(\widehat{M}, \operatorname{ext}\left(M_{\varepsilon}\right)\right)$.

Proof Fix a $(n-1)$-face $v \in S_{n-1}^{\Lambda}(\widehat{M})$ of some $\sigma \in W$. We will show that if $\operatorname{Im}(v) \cap \operatorname{int}\left(M_{\varepsilon}\right) \neq \varnothing$, then the coefficient of $v$ in $d \zeta_{L, \varepsilon}$ is null. For every $j=0, \ldots, n$ let us set $\theta_{j}^{ \pm}(v)=\left\{\sigma \in W^{ \pm} \mid \partial_{j} \sigma=v\right\}$ and $\theta_{j}(v)=\theta_{j}^{+}(v) \cup \theta_{j}^{-}(v)$. Since the coefficient of $v$ in $d \zeta_{L, \varepsilon}$ is given by

$$
\sum_{j=0}^{n}(-1)^{j}\left(\sum_{\sigma \in \theta_{j}(v)} a_{\sigma}\right),
$$

it is sufficient to prove that, under the assumption $\operatorname{Im}(v) \cap \operatorname{int}\left(M_{\varepsilon}\right) \neq \varnothing$, we have

$$
\sum_{\sigma \in \theta_{j}^{+}(v)} b_{\sigma}^{+}=\sum_{\sigma \in \theta_{j}^{-}(v)} b_{\sigma}^{-}
$$

for every $j=0, \ldots, n$.

Let us suppose $j=n$, the other cases being similar. Let $\tilde{v}$ be a fixed lift of $v$ to $\mathbb{H}^{n}$, and for every $\sigma \in \theta_{n}(v)$ let us denote by $\widetilde{\sigma}$ the unique lift of $\sigma$ such that $\partial_{n} \widetilde{\sigma}=\widetilde{v}$. By construction we have $\widetilde{v}\left(e_{i}\right) \in \widetilde{\Lambda}$ for every $i=0, \ldots, n-1$, and we denote by

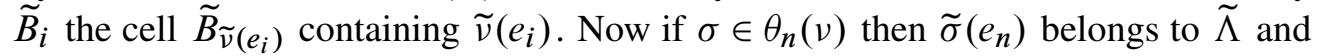
$\tilde{\sigma}\left(e_{i}\right)=\tilde{v}\left(e_{i}\right)$ for every $i=0, \ldots, n-1$. Recall that the set

$$
\Omega_{\widetilde{\sigma}}^{ \pm}=\left\{g \in G^{ \pm} \mid g\left(q_{i}\right) \in \widetilde{B}_{i} \text { for } i=0, \ldots, n-1, g\left(q_{n}\right) \in \widetilde{B}_{\widetilde{\sigma}\left(e_{n}\right)}\right\}
$$

satisfies $b_{\sigma}^{ \pm}=\mu_{G}\left(\Omega_{\tilde{\sigma}}^{ \pm}\right)$, and define

$$
\Omega_{\widetilde{v}}^{ \pm}=\left\{g \in G^{ \pm} \mid g\left(q_{i}\right) \in \widetilde{B}_{i} \text { for every } i=0, \ldots, n-1\right\} .
$$

We claim that, under the assumption $\operatorname{Im}(v) \cap \operatorname{int}\left(M_{\varepsilon}\right) \neq \varnothing$, we have

$$
\Omega_{\tilde{v}}^{ \pm}=\bigcup_{\sigma \in \theta_{n}^{ \pm}(v)} \Omega_{\widetilde{\sigma}}^{ \pm}
$$

The inclusion $\supseteq$ is obvious, while in order to get the inclusion $\subseteq$ it is sufficient to observe that if $g \in \Omega_{\tilde{v}}^{ \pm}$and $\tilde{\sigma}$ is the straight simplex with vertices in the cells containing $g\left(q_{0}\right), \ldots, g\left(q_{n}\right)$, then necessarily $\operatorname{Im}(\widetilde{\sigma}) \cap \operatorname{int}\left(\widetilde{M}_{\varepsilon}\right) \supseteq \operatorname{Im}(\widetilde{v}) \cap \operatorname{int}\left(\widetilde{M}_{\varepsilon}\right) \neq \varnothing$, so that $\sigma=\pi \circ \tilde{\sigma}$ belongs to $\theta_{n}^{ \pm}(v)$ and $g$ belongs to $\Omega \underset{\widetilde{\sigma}}{ \pm}$. 
Since the $\Omega_{\widetilde{\sigma}}^{+}$'s (resp. the $\Omega_{\widetilde{\sigma}}^{-}$'s) are pairwise disjoint, from Equation (5) we finally get

$$
\begin{aligned}
& \sum_{\sigma \in \theta_{n}^{+}(v)} b_{\sigma}^{+}=\sum_{\sigma \in \theta_{n}^{+}(v)} \mu_{G}\left(\Omega_{\tilde{\sigma}}^{+}\right)=\mu_{G}\left(\bigcup_{\sigma \in \theta_{n}^{+}(v)} \Omega_{\tilde{\sigma}}^{+}\right)=\mu_{G}\left(\Omega_{\widetilde{v}}^{+}\right), \\
& \sum_{\sigma \in \theta_{n}^{-}(v)} b_{\sigma}^{-}=\sum_{\sigma \in \theta_{n}^{-}(v)} \mu_{G}\left(\Omega_{\tilde{\sigma}}^{\bar{\sigma}}\right)=\mu_{G}\left(\bigcup_{\sigma \in \theta_{n}^{-}(v)} \Omega_{\tilde{\sigma}}^{\bar{\sigma}}\right)=\mu_{G}\left(\Omega_{\tilde{v}}^{\bar{v}}\right),
\end{aligned}
$$

so in order to conclude we are left to show that $\mu_{G}\left(\Omega_{\widetilde{v}}^{+}\right)=\mu_{G}\left(\Omega_{\widetilde{v}}^{-}\right)$. However, if $s_{\widetilde{v}} \in G^{-}$is the reflection with respect to a hyperbolic hyperplane containing $\widetilde{v}$ (such a hyperplane is unique if $\widetilde{v}$ is embedded), then $\Omega_{\widetilde{v}}^{-}=s_{\widetilde{v}} \cdot \Omega_{\widetilde{v}}^{+}$, and the conclusion follows from the left-invariance of $\mu_{G}$.

Let now $p_{*}: C_{n}\left(\widehat{M}, \operatorname{ext}\left(M_{\varepsilon}\right)\right) \rightarrow C_{n}\left(M_{\varepsilon}, \partial M_{\varepsilon}\right)$ be the map induced by the piecewise smooth retraction $p:\left(\widehat{M}, \operatorname{ext}\left(M_{\varepsilon}\right)\right) \rightarrow\left(M_{\varepsilon}, \partial M_{\varepsilon}\right)$ described in Section 2.2. The cycle

$$
\xi_{L, \varepsilon}=p_{*}\left(\zeta_{L, \varepsilon}\right)
$$

is our "efficient cycle": in order to prove Theorem 1.4, in the next section we estimate both the $L^{1}$-norm of $\xi_{L, \varepsilon}$ and the proportionality factor between the class of $\xi_{L, \varepsilon}$ in $H_{n}\left(M_{\varepsilon}, \partial M_{\varepsilon}\right)$ and the fundamental class $\left[M_{\varepsilon}\right]$ of $M_{\varepsilon}$.

\section{Proof of the main theorem}

We begin by estimating the $L^{1}$-norm of $\xi_{L, \varepsilon}$.

Lemma 4.1 We have

$$
\left\|\xi_{L, \varepsilon}\right\| \leq \operatorname{Vol}\left(N_{L}\left(M_{\varepsilon}\right)\right)
$$

Proof Since $p_{*}: C_{n}\left(\widehat{M}, \operatorname{ext}\left(M_{\varepsilon}\right)\right) \rightarrow C_{n}\left(M_{\varepsilon}, \partial M_{\varepsilon}\right)$ is norm nonincreasing we have

$$
\left\|\xi_{L, \varepsilon}\right\| \leq\left\|\zeta_{L, \varepsilon}\right\|=\sum_{\sigma \in W}\left|b_{\sigma}^{+}-b_{\sigma}^{-}\right| \leq \sum_{\sigma \in W^{+}} b_{\sigma}^{+}+\sum_{\sigma \in W^{-}} b_{\sigma}^{-} \leq \operatorname{Vol}\left(N_{L}\left(M_{\varepsilon}\right)\right),
$$

where the last inequality is due to Lemma 3.13.

\subsection{The volume form}

In order to compute the proportionality factor between $\left[\xi_{L, \varepsilon}\right]$ and $\left[M_{\varepsilon}\right]$ we would like to evaluate the Kronecker product of $\left[\xi_{L, \varepsilon}\right]$ with the volume coclass of $M_{\varepsilon}$. As usual, we first have to take care of the fact that differential forms can be integrated only on smooth simplices. So let $S_{k}^{S}(\widehat{M})\left(\operatorname{resp} . S_{k}^{s}\left(\operatorname{ext}\left(M_{\varepsilon}\right)\right)\right)$ be the set of smooth simplices with values in $\widehat{M}$ (resp. in $\left.\operatorname{ext}\left(M_{\varepsilon}\right)\right)$, let $C_{k}^{s}(\widehat{M})\left(\operatorname{resp} . C_{k}^{s}\left(\operatorname{ext}\left(M_{\varepsilon}\right)\right)\right)$ be the free $\mathbb{R}$-module generated by $S_{k}^{s}(\widehat{M})$ (resp. by $S_{k}^{s}\left(\operatorname{ext}\left(M_{\varepsilon}\right)\right)$ ) and let us set $C_{k}^{s}\left(\widehat{M}, \operatorname{ext}\left(M_{\varepsilon}\right)\right)=C_{k}^{s}(\widehat{M}) / C_{k}^{s}\left(\operatorname{ext}\left(M_{\varepsilon}\right)\right)$. A standard result of differential topology (see eg Lee [18]) ensures that a chain map $\operatorname{sm}_{*}: C_{*}(\widehat{M}) \rightarrow C_{*}^{s}(\widehat{M})$ exists such that 
(1) $\operatorname{sm}_{k}(\sigma) \in S_{k}^{s}(\widehat{M})$ for every $\sigma \in S_{k}(\widehat{M})$ and $\operatorname{sm}_{k}(\sigma) \in S_{k}^{s}\left(\operatorname{ext}\left(M_{\varepsilon}\right)\right)$ for every $\sigma \in S_{k}\left(\operatorname{ext}\left(M_{\varepsilon}\right)\right)$;

(2) $\mathrm{sm}_{*}$ restricts to the identity of $C_{*}^{s}\left(M_{\varepsilon}\right)$;

(3) if $j_{*}: C_{*}^{S}\left(M_{\varepsilon}, \partial M_{\varepsilon}\right) \rightarrow C_{*}\left(M_{\varepsilon}, \partial M_{\varepsilon}\right)$ is induced by the natural inclusion, then $\mathrm{sm}_{*}$ induces a map $C_{*}\left(M_{\varepsilon}, \partial M_{\varepsilon}\right) \rightarrow C_{*}^{s}\left(M_{\varepsilon}, \partial M_{\varepsilon}\right)$, which will still be denoted by $\mathrm{sm}_{*}$, such that $j_{*} \circ \mathrm{sm}_{*}$ is homotopic to the identity of $C_{*}\left(M_{\varepsilon}, \partial M_{\varepsilon}\right)$.

We fix an orientation on $\widehat{M}$ (whence on $M_{\varepsilon}$ ) by requiring that the fixed covering $\pi: \mathbb{H}^{n} \rightarrow \widehat{M}$ is orientation-preserving, and we denote by $\omega$ the volume differential form on $\widehat{M}$. Since the retraction $p: \widehat{M} \rightarrow M_{\varepsilon}$ defined in Section 2.2 is piecewise smooth, for every $\sigma \in S_{n}(\widehat{M})$ it makes sense to integrate $\omega$ over the composition of $\operatorname{sm}_{n}(\sigma)$ with $p$. We then define $\Omega_{\widehat{M}}: C_{n}(\widehat{M}) \rightarrow \mathbb{R}$ as the linear extension of the map

$$
\begin{aligned}
S_{n}(\widehat{M}) & \longrightarrow \mathbb{R} \\
\sigma & \longmapsto \int_{p \mathrm{osm}_{n}(\sigma)} \omega .
\end{aligned}
$$

Since $\operatorname{sm}_{*}$ is a chain map, if $c \in C_{n}(\widehat{M})$ is a boundary then $\operatorname{sm}_{n}(c)$ is the boundary of a smooth $(n+1)$-chain, so $p_{*}\left(\operatorname{sm}_{n}(c)\right)$ is the boundary of a piecewise smooth $(n+1)$-chain and as a consequence of Stokes' Theorem we have $\Omega \widehat{M}(c)=0$. This proves that $\delta \Omega \widehat{M}=0$, ie that $\Omega_{\widehat{M}}$ is an absolute cocycle. Moreover, by property (1) of the smoothing operator, if $\operatorname{Im}(\sigma) \subseteq \operatorname{ext}\left(M_{\varepsilon}\right)$ then $\operatorname{Im}\left(\operatorname{sm}_{n}(\sigma)\right) \subseteq \operatorname{ext}\left(M_{\varepsilon}\right)$, so $\operatorname{Im}\left(p \circ \operatorname{sm}_{n}(\sigma)\right) \subseteq \partial M_{\varepsilon}$ and $\Omega_{\widehat{M}}(\sigma)=0$. It follows that the cochain $\Omega_{\widehat{M}}$ vanishes on $C_{n}\left(\operatorname{ext}\left(M_{\varepsilon}\right)\right)$, ie that $\Omega_{\widehat{M}}$ is in fact a relative cocycle, and defines therefore a cohomology class $\left[\Omega_{\widehat{M}}\right] \in H^{n}\left(\widehat{M}, \operatorname{ext}\left(M_{\varepsilon}\right)\right)$.

Recall now that for every topological pair $(X, Y)$ there exists a well-defined pairing (usually called Kronecker pairing) given by

$$
\langle\cdot, \cdot\rangle: H^{p}(X, Y) \times H_{p}(X, Y) \rightarrow \mathbb{R}, \quad\langle[\varphi],[c]\rangle=\varphi(c) .
$$

Let $\zeta_{L, \varepsilon} \in C_{n}\left(\widehat{M}, \operatorname{ext}\left(M_{\varepsilon}\right)\right)$ be the cycle constructed above such that $\xi_{L, \varepsilon}=p_{*}\left(\zeta_{L, \varepsilon}\right)$, and let $i:\left(M_{\varepsilon}, \partial M_{\varepsilon}\right) \rightarrow\left(\widehat{M}, \operatorname{ext}\left(M_{\varepsilon}\right)\right)$ be the inclusion.

Lemma 4.2 We have

$$
\left[\xi_{L, \varepsilon}\right]=\frac{\Omega_{\widehat{M}}\left(\zeta_{L, \varepsilon}\right)}{\operatorname{Vol}\left(M_{\varepsilon}\right)}\left[M_{\varepsilon}\right]
$$

Proof We begin by recalling that

$$
\left\langle\left[\Omega_{\widehat{M}}\right], i_{*}\left(\left[M_{\varepsilon}\right]\right)\right\rangle=\operatorname{Vol}\left(M_{\varepsilon}\right) .
$$

In fact, if $\varphi: \Delta_{n} \rightarrow M_{\varepsilon}$ is a positively-oriented smooth embedding, then by the very definitions we have that $\Omega_{\widehat{M}}\left(i_{*}(\varphi)\right)$ equals the hyperbolic volume of $\operatorname{Im}(\varphi)$. We 
may now represent the fundamental class $\left[M_{\varepsilon}\right] \in H_{n}\left(M_{\varepsilon}, \partial M_{\varepsilon}\right)$ by a finite sum of positively-oriented embeddings $\varphi_{i}: \Delta_{n} \rightarrow T_{i}, i \in I$, where $\left\{T_{i}\right\}_{i \in I}$ is a finite smooth triangulation of $M_{\varepsilon}$. So

$$
\left\langle\left[\Omega_{\widehat{M}}\right], i_{*}\left(\left[M_{\varepsilon}\right]\right)\right\rangle=\Omega_{\widehat{M}}\left(i_{*}\left(\sum_{i \in I} \varphi_{i}\right)\right)=\sum_{i \in I} \operatorname{Vol}\left(T_{i}\right)=\operatorname{Vol}\left(M_{\varepsilon}\right) .
$$

Since $H_{n}\left(\widehat{M}, \operatorname{ext}\left(M_{\varepsilon}\right)\right) \cong H_{n}\left(M_{\varepsilon}, \partial M_{\varepsilon}\right) \cong \mathbb{R}$, this readily implies

$$
\left[\zeta_{L, \varepsilon}\right]=\frac{\left\langle\left[\Omega_{\widehat{M}}\right],\left[\zeta_{L, \varepsilon}\right]\right\rangle}{\operatorname{Vol}\left(M_{\varepsilon}\right)} \cdot i_{*}\left(\left[M_{\varepsilon}\right]\right)=\frac{\Omega \widehat{M}\left(\zeta_{L, \varepsilon}\right)}{\operatorname{Vol}\left(M_{\varepsilon}\right)} \cdot i_{*}\left(\left[M_{\varepsilon}\right]\right)
$$

whence

$$
\left[\xi_{L, \varepsilon}\right]=p_{*}\left(\left[\zeta_{L, \varepsilon}\right]\right)=\frac{\Omega_{\widehat{M}}\left(\zeta_{L, \varepsilon}\right)}{\operatorname{Vol}\left(M_{\varepsilon}\right)} p_{*}\left(i_{*}\left(\left[M_{\varepsilon}\right]\right)\right)=\frac{\Omega_{\widehat{M}}\left(\zeta_{L, \varepsilon}\right)}{\operatorname{Vol}\left(M_{\varepsilon}\right)}\left[M_{\varepsilon}\right]
$$

In order to estimate the proportionality coefficient between $\left[\xi_{L, \varepsilon}\right]$ and $\left[M_{\varepsilon}\right]$ we are therefore left to compute $\Omega \widehat{M}\left(\zeta_{L, \varepsilon}\right)$. From now on, we denote by $L_{0}$ the positive constant provided by Lemma 3.6. Also recall that $R_{L}$ and $V_{L}$ were introduced in Definition 3.5.

Corollary 4.3 Suppose $L \geq L_{0}$. For every $\sigma \in W$ we have $\Omega \widehat{M}\left(a_{\sigma} \sigma\right) \geq 0$. Moreover, if $\sigma \in W_{\text {int }}^{+}\left(\right.$resp. $\sigma \in W_{\text {int }}^{-}$), then $\Omega_{\widehat{M}}(\sigma) \geq V_{L}\left(\operatorname{resp} . \Omega_{\widehat{M}}(\sigma) \leq-V_{L}\right)$.

Proof Since the covering projection $\pi: \mathbb{H}^{n} \rightarrow \widehat{M}$ is an orientation-preserving local isometry, if $\sigma$ is any straight simplex in $\widehat{M}$ and $\widetilde{\sigma}$ is any lift of $\sigma$ in $\mathbb{H}^{n}$, then $\Omega \widehat{M}(\sigma)$ is the signed volume of the portion of $\operatorname{Im}(\widetilde{\sigma})$ contained in $\widetilde{M}_{\varepsilon}$.

Now, if $\sigma \in W^{+}$and $\tilde{\sigma}$ is any lift of $\sigma$, then by construction we have $\widetilde{\sigma} \in R_{L}$, so by Lemma 3.6 the simplex $\widetilde{\sigma}$ is positively-oriented and $\operatorname{Vol}_{\text {alg }}(\widetilde{\sigma}) \geq V_{L}$. This implies that the signed volume of the portion of $\operatorname{Im}(\widetilde{\sigma})$ contained in $\widetilde{M}_{\varepsilon}$ is nonnegative, so $\Omega_{\widehat{M}}(\sigma) \geq 0$. Moreover, if $\sigma \in W_{\text {int }}^{+}$, then $\operatorname{Im}(\widetilde{\sigma}) \subseteq \operatorname{int}\left(\widetilde{M}_{\mathcal{E}}\right)$, so $\Omega_{\widehat{M}}(\sigma)=$ $\operatorname{Vol}_{\text {alg }}(\tilde{\sigma}) \geq V_{L}$.

On the other hand, it is easily seen that if $\tilde{\sigma}$ is any lift of a simplex $\sigma \in W^{-}$and $g \in G^{-}$is any orientation-reversing isometry of $\mathbb{H}^{n}$, then $g \cdot \widetilde{\sigma} \in R_{L}$. Therefore $\operatorname{Vol}_{\text {alg }}(g \cdot \widetilde{\sigma}) \geq V_{L}$ and $g \cdot \widetilde{\sigma}$ is positively-oriented, so $\operatorname{Vol}_{\text {alg }}(\widetilde{\sigma})=-\operatorname{Vol}_{\text {alg }}(g \cdot \tilde{\sigma}) \leq-V_{L}$, and $\widetilde{\sigma}$ is negatively-oriented. Just as before, this readily implies that $\Omega_{\widehat{M}}(\sigma) \leq 0$, and that $\Omega \widehat{M}(\sigma) \leq-V_{L}$ whenever $\sigma \in W_{\text {int }}^{-}$.

In order to conclude we are left to show that $\Omega_{\widehat{M}}\left(a_{\sigma} \sigma\right) \geq 0$ for every $\sigma \in W$. As observed above, if $\sigma \in W^{+}$(resp. $\sigma \in W^{-}$), then every lift of $\sigma$ is positively-oriented 
(resp. negatively-oriented), and this readily implies that $W^{+} \cap W^{-}=\varnothing$. Therefore, if $\sigma \in W$, then either $\sigma \in W^{+}$, and $\Omega_{\widehat{M}}\left(a_{\sigma} \sigma\right)=b_{\sigma}^{+} \Omega_{\widehat{M}}(\sigma) \geq 0$, or $\sigma \in W^{-}$, and again $\Omega_{\widehat{M}}\left(a_{\sigma} \sigma\right)=-b_{\sigma}^{-} \Omega_{\widehat{M}}(\sigma) \geq 0$.

\subsection{The final step}

We are now ready to provide the required estimate on $\Omega_{\widehat{M}}\left(\zeta_{L, \varepsilon}\right)$.

Proposition 4.4 Suppose $L \geq L_{0}$. Then

$$
\Omega_{\widehat{M}}\left(\zeta_{L, \varepsilon}\right) \geq V_{L} \cdot \operatorname{Vol}\left(M_{\varepsilon} \backslash N_{L+3}\left(\partial M_{\varepsilon}\right)\right) .
$$

Proof We decompose $\zeta_{L, \varepsilon}$ as the sum $\zeta_{L, \varepsilon}=\zeta_{\text {int }}^{+}+\zeta_{\text {int }}^{-}+\zeta_{\text {ext }}$, where

$$
\zeta_{\mathrm{int}}^{+}=\sum_{\sigma \in W_{\mathrm{int}}^{+}} b_{\sigma}^{+} \sigma, \quad \zeta_{\mathrm{int}}^{-}=-\sum_{\sigma \in W_{\mathrm{int}}^{-}} b_{\sigma}^{-} \sigma, \quad \zeta_{\mathrm{ext}}=\sum_{\sigma \in W_{\mathrm{ext}}} a_{\sigma} \sigma .
$$

By Corollary 4.3 and Lemma 3.13 we get

$$
\begin{array}{ll}
\Omega_{\widehat{M}}\left(\zeta_{\text {int }}^{+}\right) \geq\left(\sum_{\sigma \in W_{\text {int }}^{+}} b_{\sigma}^{+}\right) V_{L} & \geq\left(\operatorname{Vol}\left(M_{\varepsilon} \backslash N_{L+3}\left(\partial M_{\varepsilon}\right)\right) \cdot V_{L}\right) / 2, \\
\Omega_{\widehat{M}}\left(\zeta_{\text {int }}^{-}\right) \geq\left(\sum_{\sigma \in W_{\text {int }}^{-}} b_{\sigma}^{-}\right) V_{L} & \geq\left(\operatorname{Vol}\left(M_{\varepsilon} \backslash N_{L+3}\left(\partial M_{\varepsilon}\right)\right) \cdot V_{L}\right) / 2, \\
\Omega_{\widehat{M}}\left(\zeta_{\text {ext }}\right)=\left(\sum_{\sigma \in W_{\text {ext }}} \Omega_{\widehat{M}}\left(a_{\sigma} \sigma\right)\right) \geq 0,
\end{array}
$$

whence the conclusion by linearity of $\Omega_{\widehat{M}}$.

Corollary 4.5 We have

$$
\frac{\operatorname{Vol}(M)}{\|\bar{M}\|} \geq \frac{\operatorname{Vol}\left(M_{\varepsilon} \backslash N_{L+3}\left(\partial M_{\varepsilon}\right)\right)}{\operatorname{Vol}\left(N_{L}\left(M_{\varepsilon}\right)\right)} \cdot V_{L} .
$$

Proof Since $\bar{M}$ is diffeomorphic to $M_{\varepsilon}$ we have $\|\bar{M}\|=\left\|M_{\varepsilon}\right\|=\left\|\left[M_{\varepsilon}\right]\right\|$. Thus from Lemma 4.1, Lemma 4.2 and Proposition 4.4 we get

$$
\|\bar{M}\|=\left\|\frac{\operatorname{Vol}\left(M_{\varepsilon}\right)}{\Omega_{\widehat{M}}\left(\zeta_{L, \varepsilon}\right)}\left[\xi_{L, \varepsilon}\right]\right\| \leq\left|\frac{\operatorname{Vol}\left(M_{\varepsilon}\right)}{\Omega_{\widehat{M}}\left(\zeta_{L, \varepsilon}\right)}\right|\left\|\xi_{L, \varepsilon}\right\| \leq \frac{\operatorname{Vol}\left(N_{L}\left(M_{\varepsilon}\right)\right) \cdot \operatorname{Vol}\left(M_{\varepsilon}\right)}{V_{L} \cdot \operatorname{Vol}\left(M_{\varepsilon} \backslash N_{L+3}\left(\partial M_{\varepsilon}\right)\right)}
$$

Since $\operatorname{Vol}(M) \geq \operatorname{Vol}\left(M_{\varepsilon}\right)$, this readily implies the conclusion.

In order to conclude we now need some estimates on the volume of $L$-neighbourhoods of geodesic hypersurfaces in hyperbolic manifolds. For $t \geq 0$ let

$$
g(t)=2 \int_{0}^{L} \cosh ^{n-1}(t) \mathrm{d} t .
$$


An easy computation (see eg Basmajian [1]) shows that if $A$ is an embedded totally geodesic hypersurface in a hyperbolic $n$-manifold $X$, then the $n$-dimensional volume of any embedded tubular $t$-neighbourhood of $A$ in $X$ is given by $g(t) \cdot \operatorname{Vol}(A)$.

Lemma 4.6 For every $L>0$ we have

$$
\lim _{\varepsilon \rightarrow 0} \operatorname{Vol}\left(N_{L}\left(\partial M_{\varepsilon}\right)\right) \leq g(L) \cdot \operatorname{Vol}(\partial M) .
$$

Proof Recall that $\partial M_{\varepsilon}=\partial M \cup T_{\varepsilon}$, where $T_{\varepsilon}$ is the union of the boundaries of the deleted cusps. Therefore $N_{L}\left(\partial M_{\varepsilon}\right)=N_{L}(\partial M) \cup N_{L}\left(T_{\varepsilon}\right)$ and it is easily seen that $\lim _{\varepsilon \rightarrow 0} \operatorname{Vol}\left(N_{L}\left(T_{\varepsilon}\right)\right)=0$, whence $\lim _{\varepsilon \rightarrow 0} \operatorname{Vol}\left(N_{L}\left(\partial M_{\varepsilon}\right)\right)=\operatorname{Vol}\left(N_{L}(\partial M)\right)$.

Let now $B$ be a connected component of $\partial M$ and let $X \rightarrow \widehat{M}$ be the Riemannian covering associated to the image of $\pi_{1}(B)$ into $\pi_{1}(M)$. Then $X$ is diffeomorphic to $B \times(-\infty,+\infty)$ and contains a totally geodesic hypersurface $B \times\{0\}$ isometric to $B$. The $L$-neighbourhood of $B \times\{0\}$ in $X$ is embedded and has therefore volume $g(L) \cdot \operatorname{Vol}(B)$. Since the projection $X \rightarrow \widehat{M}$ is a local isometry and maps (possibly not injectively) such a neighbourhood onto $N_{L}(B) \subseteq \widehat{M}$, it follows that $\operatorname{Vol}\left(N_{L}(B)\right) \leq$ $g(L) \cdot \operatorname{Vol}(B)$. If $B_{1}, \ldots, B_{k}$ are the components of $\partial M$ we then have

$$
\operatorname{Vol}\left(N_{L}(\partial M)\right) \leq \sum_{i=1}^{k} \operatorname{Vol}\left(N_{L}\left(B_{i}\right)\right) \leq g(L)\left(\sum_{i=1}^{k} \operatorname{Vol}\left(B_{i}\right)\right)=g(L) \cdot \operatorname{Vol}(\partial M),
$$

whence the conclusion.

Let us put the estimate of Lemma 4.6 into inequality (6). We have

$$
\operatorname{Vol}\left(M_{\varepsilon} \backslash N_{L+3}\left(\partial M_{\varepsilon}\right)\right) \geq \operatorname{Vol}\left(M_{\varepsilon}\right)-\operatorname{Vol}\left(N_{L+3}\left(\partial M_{\varepsilon}\right)\right),
$$

so since $\lim _{\varepsilon \rightarrow 0} \operatorname{Vol}\left(M_{\varepsilon}\right)=\operatorname{Vol}(M)$ we get

$$
\lim _{\varepsilon \rightarrow 0} \operatorname{Vol}\left(M_{\varepsilon} \backslash N_{L+3}\left(\partial M_{\varepsilon}\right)\right) \geq \operatorname{Vol}(M)-g(L+3) \cdot \operatorname{Vol}(\partial M) .
$$

In the same way we get $\lim _{\varepsilon \rightarrow 0} \operatorname{Vol}\left(N_{L}\left(M_{\varepsilon}\right)\right) \leq \operatorname{Vol}(M)+g(L) \cdot \operatorname{Vol}(\partial M)$. Therefore, if $r=\operatorname{Vol}(\partial M) / \operatorname{Vol}(M)$, then passing to the limit in the right-hand side of (6) we obtain

$$
\frac{\operatorname{Vol}(M)}{\|\bar{M}\|} \geq \frac{1-r \cdot g(L+3)}{1+r \cdot g(L)} \cdot V_{L} .
$$

Let now $\eta<v_{n}$ be given. By Lemma 3.6 there exists $L_{1} \geq L_{0}$ (only depending on $n$ and $\eta$ ) such that $V_{L_{1}}>v_{n}-\eta / 2$. Since

$$
\lim _{r \rightarrow 0}\left(1-r \cdot g\left(L_{1}+3\right)\right) /\left(1+r \cdot g\left(L_{1}\right)\right)=1 \text {, }
$$


there exists $k>0$ (only depending on $L_{1}$, that is on $n$ and $\eta$ ) such that

$$
\left(1-r \cdot g\left(L_{1}+3\right)\right) /\left(1+r \cdot g\left(L_{1}\right)\right)>\left(v_{n}-\eta\right) /\left(v_{n}-\eta / 2\right)
$$

for every $r \leq k$. Inequality (7) with $L=L_{1}$ now shows that if $r=\operatorname{Vol}(\partial M) / \operatorname{Vol}(M) \leq k$ then

$$
\frac{\operatorname{Vol}(M)}{\|\bar{M}\|} \geq \frac{v_{n}-\eta}{v_{n}-\eta / 2}\left(v_{n}-\eta / 2\right)=v_{n}-\eta,
$$

and this concludes the proof of Theorem 1.4.

\section{References}

[1] A Basmajian, Tubular neighborhoods of totally geodesic hypersurfaces in hyperbolic manifolds, Invent. Math. 117 (1994) 207-225 MR1273264

[2] R Benedetti, C Petronio, Lectures on hyperbolic geometry, Universitext, Springer, Berlin (1992) MR1219310

[3] M Bucher-Karlsson, Simplicial volume of locally symmetric spaces covered by $\mathrm{SL}_{3} \mathbb{R} / \mathrm{SO}(3)$, Geom. Dedicata 125 (2007) 203-224 MR2322549

[4] M Bucher-Karlsson, The simplicial volume of closed manifolds covered by $\mathbb{H}^{2} \times \mathbb{H}^{2}$, J. Topol. 1 (2008) 584-602 MR2417444

[5] S Francaviglia, Hyperbolic volume of representations of fundamental groups of cusped 3-manifolds, Int. Math. Res. Not. (2004) 425-459 MR2040346

[6] R Frigerio, Commensurability of hyperbolic manifolds with geodesic boundary, Geom. Dedicata 118 (2006) 105-131 MR2239451

[7] M Gromov, Volume and bounded cohomology, Inst. Hautes Études Sci. Publ. Math. (1982) 5-99 (1983) MR686042

[8] M Gromov, I Piatetski-Shapiro, Nonarithmetic groups in Lobachevsky spaces, Inst. Hautes Études Sci. Publ. Math. (1988) 93-103 MR932135

[9] U Haagerup, HJ Munkholm, Simplices of maximal volume in hyperbolic n-space, Acta Math. 147 (1981) 1-11 MR631085

[10] S K Hansen, Measure homology, Math. Scand. 83 (1998) 205-219 MR1673914

[11] D L Johnson, A short proof of the uniqueness of Haar measure, Proc. Amer. Math. Soc. 55 (1976) 250-251 MR0396914

[12] D Jungreis, Chains that realize the Gromov invariant of hyperbolic manifolds, Ergodic Theory Dynam. Systems 17 (1997) 643-648 MR1452185

[13] S Kojima, Polyhedral decomposition of hyperbolic manifolds with boundary, Proc. Workshop Pure Math. 10 (1990) 37-57 
[14] S Kojima, Geometry of hyperbolic 3-manifolds with boundary, Kodai Math. J. 17 (1994) 530-537 MR1296924 Workshop on Geometry and Topology (Hanoi, 1993)

[15] T Kuessner, Efficient fundamental cycles of cusped hyperbolic manifolds, Pacific J. Math. 211 (2003) 283-313 MR2015738

[16] T Kuessner, Proportionality principle for cusped manifolds, Arch. Math. (Brno) 43 (2007) 485-490 MR2381790

[17] J-F Lafont, B Schmidt, Simplicial volume of closed locally symmetric spaces of noncompact type, Acta Math. 197 (2006) 129-143 MR2285319

[18] J M Lee, Introduction to smooth manifolds, Graduate Texts in Math. 218, Springer, New York (2003) MR1930091

[19] C Löh, Measure homology and singular homology are isometrically isomorphic, Math. Z. 253 (2006) 197-218 MR2206643

[20] C Löh, R Sauer, Degree theorems and Lipschitz simplicial volume for nonpositively curved manifolds of finite volume, J. Topol. 2 (2009) 193-225 MR2499443

[21] N Peyerimhoff, Simplices of maximal volume or minimal total edge length in hyperbolic space, J. London Math. Soc. (2) 66 (2002) 753-768 MR1934304

[22] J G Ratcliffe, Foundations of hyperbolic manifolds, Graduate Texts in Math. 149, Springer, New York (1994) MR1299730

[23] W P Thurston, The geometry and topology of three-manifolds, Princeton Univ. Math. Dept. Lecture Notes (1979) Available at http://msri.org/publications/books/ gt $3 \mathrm{~m} /$

[24] A Weil, L'intégration dans les groupes topologiques et ses applications, Actual. Sci. Ind. 869, Hermann et Cie., Paris (1940) MR0005741 [This book has been republished by the author at Princeton, 1941.]

[25] A Zastrow, On the (non)-coincidence of Milnor-Thurston homology theory with singular homology theory, Pacific J. Math. 186 (1998) 369-396 MR1663826

Dipartimento di Matematica "L Tonelli", Università di Pisa Largo B Pontecorvo 5, I-56127 Pisa, Italy

frigerio@dm.unipi.it, pagliantini@mail.dm.unipi.it http://www.dm.unipi.it/ frigerio/

Received: 7 November 2009 Revised: 14 March 2010 